\title{
Investigation of the magnetic phase transition and magnetocaloric properties of the $\mathrm{Mn}_{2} \mathrm{FeSbO}_{6}$ ilmenite
}

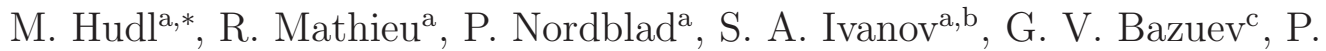 \\ Lazor $^{\mathrm{d}}$ \\ ${ }^{a}$ Department of Engineering Sciences, Uppsala University, Box 534, SE-751 21 Uppsala \\ Sweden \\ ${ }^{b}$ Department of Inorganic Materials, Karpov' Institute of Physical Chemistry, \\ Vorontsovo pole, 10 105064, Moscow K-64, Russia \\ ${ }^{c}$ Institute of Solid-State Chemistry, Ural Branch of the Russian Academy of Science, \\ 620999 Ekaterinburg, GSP-145, Russia \\ ${ }^{d}$ Department of Earth Sciences, Uppsala University, Villavägen 16, SE-752 36 Uppsala \\ Sweden
}

\begin{abstract}
The magnetic phase transition and magnetocaloric properties of both mineral and synthetic melanostibite $\mathrm{Mn}_{2} \mathrm{FeSbO}_{6}$ with ilmenite-type structure have been studied. $\mathrm{Mn}_{2} \mathrm{FeSbO}_{6}$ orders ferrimagnetically below $270 \mathrm{~K}$ and is found to undergo a second-order magnetic phase transition. The associated magnetic entropy change was found to be $1.7 \mathrm{~J} / \mathrm{kgK}$ for the mineral and $1.8 \mathrm{~J} / \mathrm{kgK}$ synthetic melanostibite for $5 \mathrm{~T}$ field change. For the synthetic $\mathrm{Mn}_{2} \mathrm{FeSbO}_{6}$ the adiabatic temperature change was estimated from magneticand specific heat measurements and amounts to $0.2 \mathrm{~K}$ in $1 \mathrm{~T}$ field change. Perspectives of the promising functional properties of $\mathrm{Mn}_{2} \mathrm{FeSbO}_{6}$-based materials are discussed.
\end{abstract}

Keywords: Melanostibite, magnetocaloric properties, magnetic phase transition

\section{Introduction}

It has been shown that layered magnetic systems can give rise to interesting functional properties e.g. an enhanced magnetocaloric effect such as in

\footnotetext{
${ }^{*}$ Corresponding author

Email address: matthias.hudl@angstrom.uu.se (M. Hudl)
} 
$\mathrm{Gd}_{5}\left(\mathrm{Si}_{1-x} \mathrm{Ge}_{x}\right)_{4}$ and $(\mathrm{Mn}, \mathrm{Fe})_{2}(\mathrm{P}, \mathrm{Si})$ compounds [1, 2]. The magnetocaloric effect is associated with the temperature change of a magnetic material due to a change of the magnetization under applied magnetic fields. Magnetocaloric materials are intensively studied because of the potential use for cooling and heating applications. The primary advantage of magnetocaloric materials, as opposed to conventional vapor-cycle based technology, is the two orders of magnitude larger entropy density in a solid near its transition temperature compared to a gaseous medium and an efficiency of the magnetic cycle closer to the Carnot limit [3]. Nevertheless, to use that potential in an environmentally friendly and energy efficient way, materials not based on expensive, toxic and rare elements are needed.

Melanostibite is a mineral found in Sjögruvan (Örebro, Sweden) and was first described by Lars Johan Igelström [4] in 1892. The name melanostibite reflects on the color (melanos $=$ black in greek) and composition (stibium $=$ antimony in latin) of the material. Melanostibite is a Mn-Sb-oxide with chemical formula $\mathrm{Mn}_{2} \mathrm{FeSbO}_{6}$ consisting of abundant elements and has a hexagonal crystal structure with space group $R \overline{3}$, which is an isostructural to the ilmenite-pyrofanite group [5]. The octahedral representation of ilmenite-type structure is shown in Figure 1. In this structure $\mathrm{Mn}$ and $\mathrm{Fe} / \mathrm{Sb}$ are ordered in layers along the $c$-axis, whereas $\mathrm{Fe}$ and $\mathrm{Sb}$ are completely disordered within a $\mathrm{Fe} / \mathrm{Sb}$ layer. Recently, it has been shown that $\mathrm{Mn}_{2} \mathrm{FeSbO}_{6}$ mineral exhibits a ferrimagnetic ordering near $270 \mathrm{~K}$ brought forth by the $\mathrm{Mn}^{2+}$ and $\mathrm{Fe}^{3+}$ ions in the structure [6]. The magnetic transition in $\mathrm{Mn}_{2} \mathrm{FeSbO}_{6}$ is evident from magnetization measurements in low magnetic fields. Nevertheless, its order and the magnetic properties in higher magnetic fields have not been studied yet. A magnetic phase transition close to room temperature and the layered magnetic structure suggesting that this material could give rise to interesting magnetocaloric properties near room temperature. Magnetocaloric oxide materials with perovskite-type structure have been intensively studied [7, 8, 9]. To this date there is only a small number of known materials with ilmenitetype structure and a magnetic transition close to room temperature [10]. No study of their magnetocaloric properties have been reported.

In this article we report on the magnetic phase transition and the magnetocaloric properties of both mineral and synthetic $\mathrm{Mn}_{2} \mathrm{FeSbO}_{6}$ with ilmenitetype structure. Our results are based on magnetic- and specific heat measurements. The magnetic entropy changes $\Delta S_{M}$ for the mineral (synthetic) melanostibite sample was found to be $1.7(1.8) \mathrm{J} / \mathrm{kgK}$ and $0.51(0.46) \mathrm{J} / \mathrm{kgK}$ for field changes of 5 and 1 Tesla, respectively. Although $\Delta S_{M}$ is relatively 


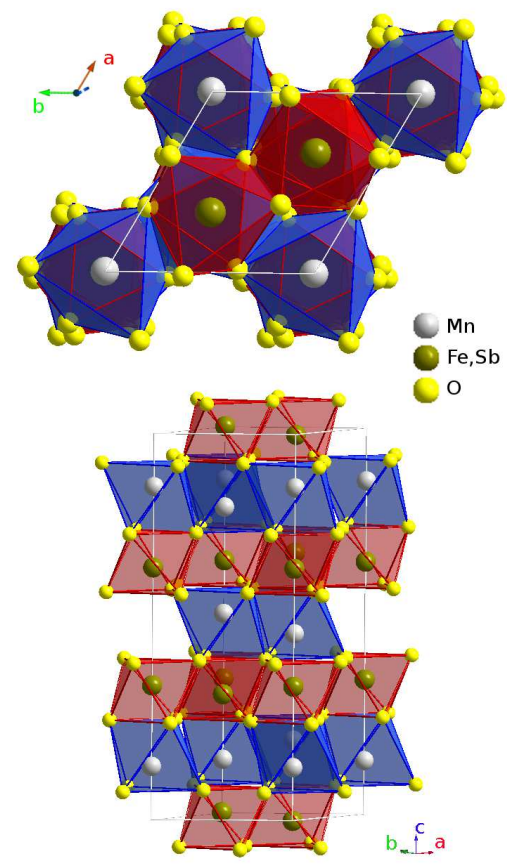

Figure 1: (Color online) Octahedral representation of the melanostibite crystal lattice with ilmenite structure; along $c$-axis (upper panel) and in $a b$-plane direction (lower panel).

small compared to reference magnetocaloric materials such as $\mathrm{Gd}$, we believe that our results indicate that new materials based on ferri-/ferromagnetic ilmenites such as $\mathrm{Mn}_{2} \mathrm{FeSbO}_{6}$ with improved magnetocaloric properties near room-temperature could be designed.

\section{Experimental methods}

Synthetic $\mathrm{Mn}_{2} \mathrm{FeSbO}_{6}$ samples were fabricated using conventional solid state reaction (including terminatory sintering at temperatures between 1300 and $1350{ }^{\circ} \mathrm{C}$ ) and thermobaric treatment under a pressure of $3 \mathrm{GPa}$ at 1000 ${ }^{\circ} \mathrm{C}$ for $30 \mathrm{~min}$. Detailed synthesis conditions are reported in Ref. Mathieu et al. [6] and Bazuev et al. [11]. The phase composition of the synthetic samples was studied using powder x-ray diffraction (XRD) method on a D8 Bruker diffractometer with $\mathrm{CuK} \alpha 1$ radiation. The ICDD PDF4 database of standard powder patterns (ICDD, USA, Release 2009) was used to identify possible impurity phases. The cation stoichiometry of the mineral and synthetic samples was checked using microprobe EDX analysis (average of 


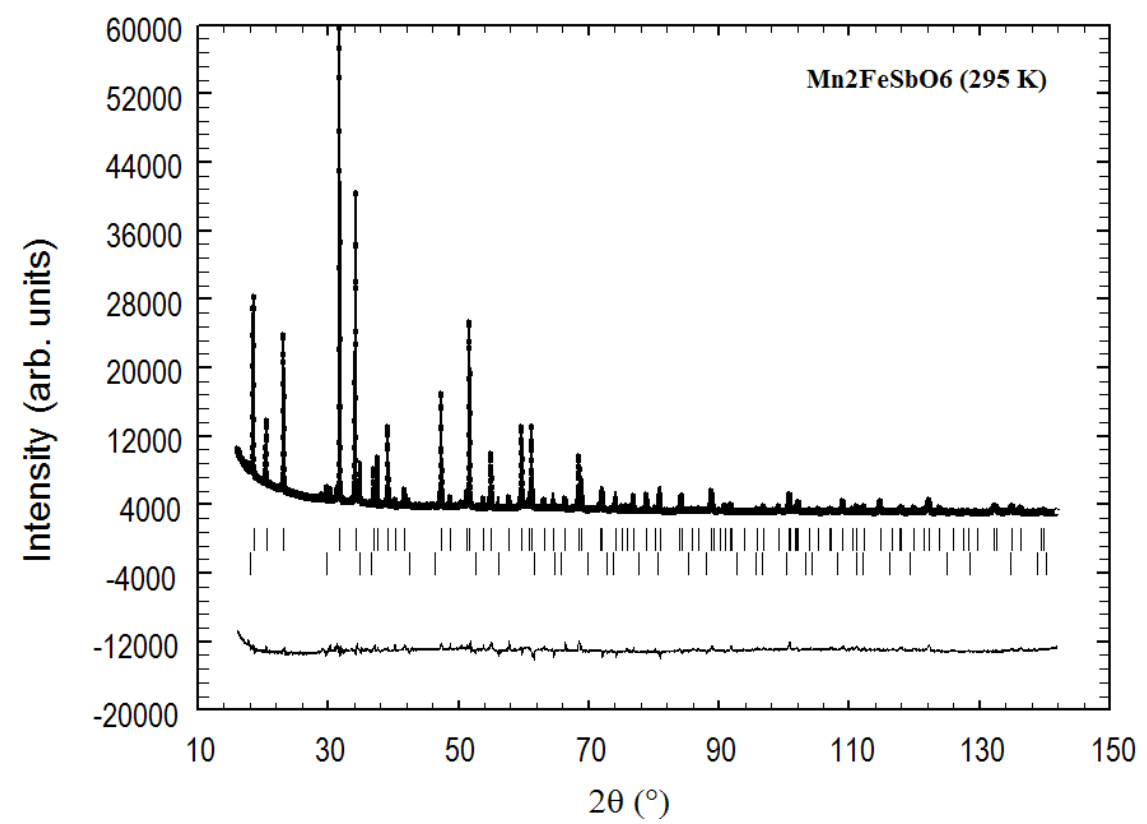

Figure 2: X-ray diffraction pattern and Rietveld refinement $\left(\mathrm{R}_{p}=5.64 \%, \mathrm{R}_{w p}=7.32 \%\right.$, and $\mathrm{R}_{\beta}=3.18 \%$ ) for synthetic melanostibite at $295 \mathrm{~K}$.

20 points). Magnetization measurements for all samples were done using a SQUID magnetometer (MPMS-XL) from Quantum Design Inc (QD). For the magnetization measurements of the natural sample several small $(\approx 10$ $500 \mu \mathrm{m}$ ) crystals of the mineral melanostibite were assembled together(total weight of $3.7 \mathrm{mg})$. Specific heat measurements were performed using a relaxation method on a Physical Properties Measurement System (QD PPMS $6000)$.

Incidentally, the melanostibite mineral was found ten years after the discovery of the magnetocaloric effect by E. Warburg in 1881 [12]. The magnetocaloric effect is related to the coupling of the spin system with the lattice vibrations of the material and can be represented by the change of magnetic part of the total entropy $\Delta S_{M}$.

For an adiabatic magnetization process the change of the magnetic entropy part causes an identical change in the lattice part of the total entropy evident as a change in temperature $\Delta T_{a d}$ [13]. The magnetic entropy change for a magnetic material undergoing a second-order magnetic phase transition can indeed be derived from Maxwell-relations [14]. Hence, $\Delta S_{M}$ for a 

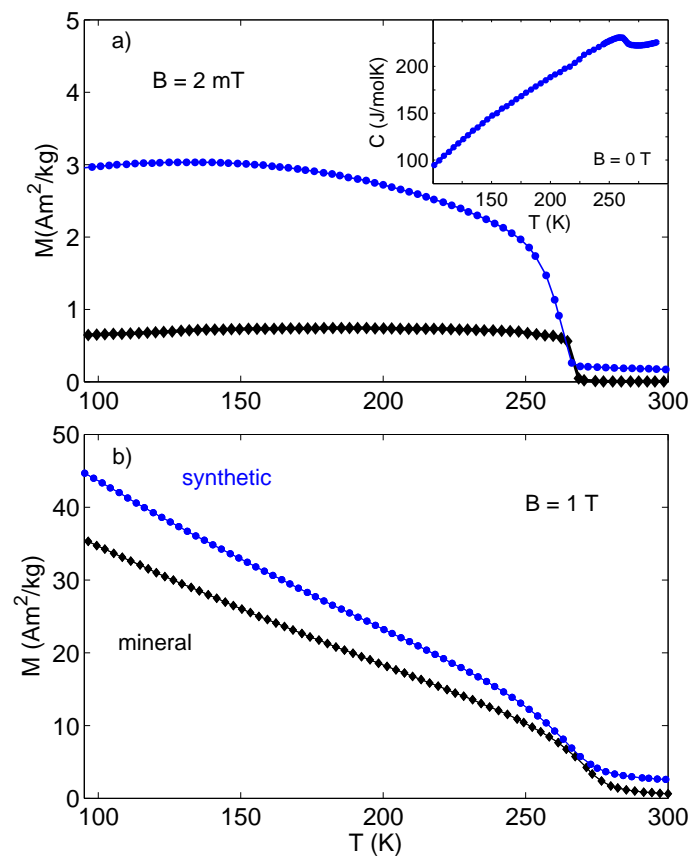

Figure 3: (Color online) Field-cooled magnetization data recorded on cooling (FCC) as a function of temperature for a natural mineral $(\bullet)$ and synthetic $(\bullet)$ sample melanostibite measured in a) $0.02 \mathrm{mT}$ and b) $1 \mathrm{~T}$. The inset shows the specific heat of the synthetic sample measured in zero magnetic field.

magnetic field change from $H_{i}$ to $H_{f}$ can be calculated using

$$
\Delta S_{M}=\mu_{0} \int_{H_{i}}^{H_{f}}\left(\frac{\partial M}{\partial T}\right)_{H} d H .
$$

From isothermal magnetization measurements as a function of the magnetic field, $\left.M(H)\right|_{T}, \Delta S_{M}$ was calculated using the numerical integration

$$
\Delta S_{M}(T)=\mu_{0} \sum_{i, j}\left(\frac{M_{i+1}-M_{i}}{T_{i+1}-T_{i}}\right)\left(H_{j+1}-H_{j}\right),
$$

where $i$ is the number of isothermal measurements, $\left(T=\left(T_{i+1}+T_{i}\right) / 2\right)$, and $j$ labels the magnetic field increments $\left(\Delta H=\sum_{j} H_{j}\right)$. In terms of evaluating the nature of a magnetic phase transition, a technique proposed by A. Arrott [15, 16] can be used. The field dependence of the magnetization 

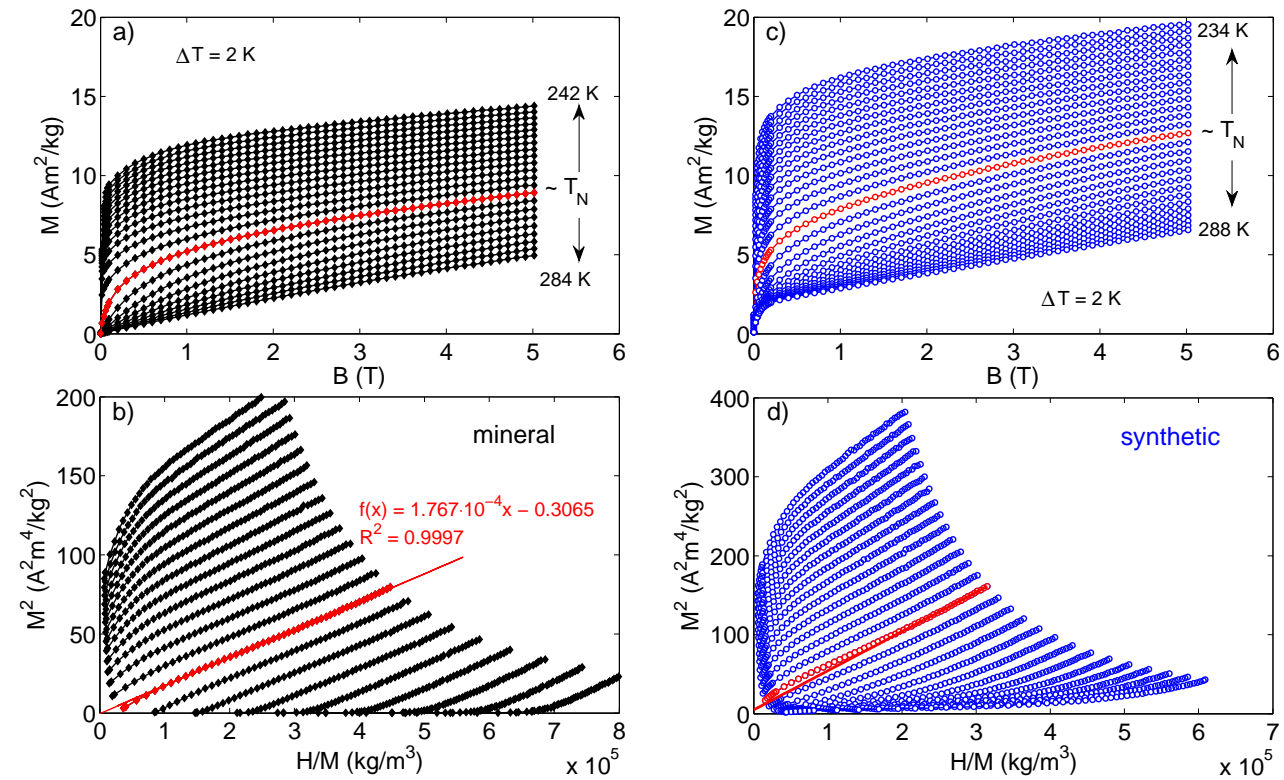

Figure 4: (Color online) Magnetization as a function of magnetic field for a) the natural mineral and c) the synthetic sample melanostibite around $T_{N}$. Corresponding Arrott plots for $b$ ) the natural mineral and d) synthetic melanostibite. A linear fit for the mineral sample near $T_{N}$ is shown in b), $\mathrm{R}^{2}$ refers to the accuracy of the least-square fitting.

can be expressed as a power series $\left(H=(1 / \chi) M+\beta M^{3}+\gamma M^{5}+\ldots\right)$ of the order parameter $M$. At the Curie point the magnetic susceptibility approaches infinity and $(1 / \chi)=0$, which should result in straight lines in the "critical region" around the phase transition when plotted as $M^{2}$ vs. $H / M$ (mean field approximation). Analyzing the slope of data plotted as $M^{2}$ vs. $H / M$ indicates the order of phase transition (Banerjee criterion) [17]. A positive slope implies a second-order-continuous phase transition, whereas an S-shaped negative slope suggests a first-order-discontinous phase transition.

\section{Results and discussion}

An X-ray diffraction pattern for synthetic melanostibite is shown in Figure 2. The main phase of the synthesized sample crystallizes in the hexagonal ilmenite-type structure (space group $\mathrm{R} \overline{3}, a=5.237(1) \AA$ and $c=14.349(2)$ $\AA$ ) similar to the single-crystal mineral of $\mathrm{Mn}_{2} \mathrm{FeSbO}_{6}$. From Rietveld refinement,with $\mathrm{R}$-factors $\mathrm{R}_{p}=5.64 \%, \mathrm{R}_{w p}=7.32 \%$, and $\mathrm{R}_{\beta}=3.18 \%$, a minor 
amount $(\approx 2 \%)$ of a secondary phase of the $\mathrm{MnFe}_{2} \mathrm{O}_{4}$ spinel, which is ferrimagnetic below $T_{N}=570 \mathrm{~K}$, was estimated.

In Figure 3, the magnetization curves for a mineral (black curve) and a synthetic sample (blue curve) of melanostibite are shown. In small magnetic fields $\left(2 \mathrm{mT}\right.$ ) both mineral and synthetic $\mathrm{Mn}_{2} \mathrm{FeSbO}_{6}$ show a sharp phase transition. The transition temperature for the mineral is found to be $\sim 268$ $\mathrm{K}$ and for the synthetic sample slightly lower at $\sim 264 \mathrm{~K}$. The low temperature magnetization for a 5 Tesla field revealed a moment of about $3.7 \mu_{B} / f$.u. for the mineral and $4.35 \mu_{B} / f . u$. (63.1 $\left.\mathrm{Am}^{2} / \mathrm{kg}\right)$ for the synthetic melanostibite at $10 \mathrm{~K}$ and is in agreement with a ferrimagnetic arrangement of $2 \mathrm{Mn}^{2+}$ and $1 \mathrm{Fe}^{3+}$ cations (all carrying $S=5 / 2,5 \mu_{B}$ ) per formula unit [6]. For a magnetic field of $1 \mathrm{~T}$, Figure $3 \mathrm{~b}$, the transition appears broader and the magnetization is increasing linearly below $T_{N}$ due to the ferrimagnetic type ordering. The inset in Figure 3 a shows the specific heat for the synthetic melanostibite in $0 \mathrm{~T}$ field.
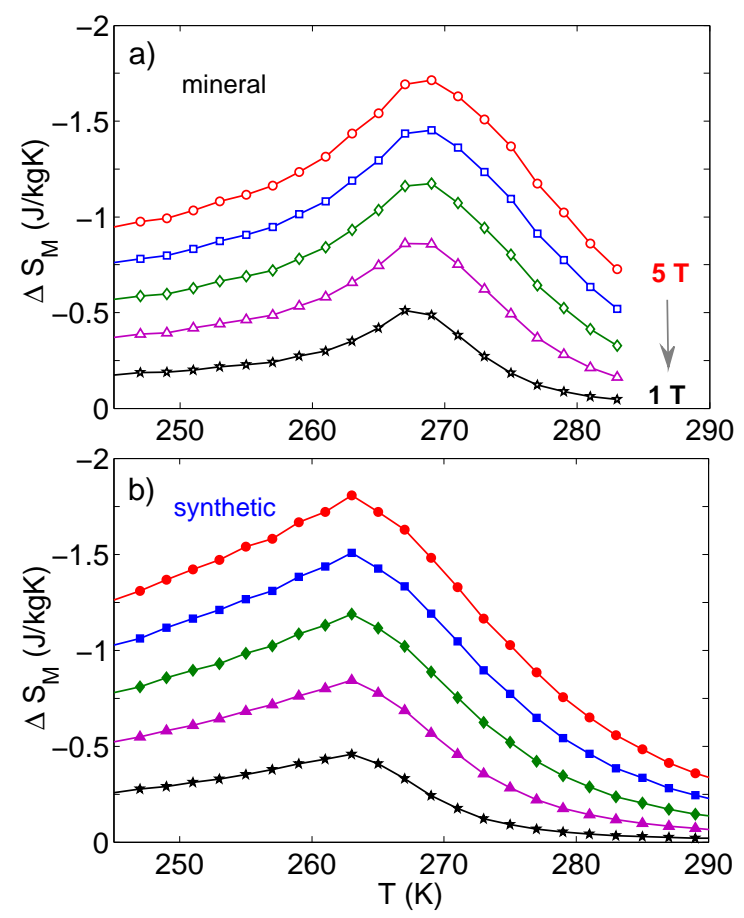

Figure 5: (Color online) Magnetic entropy change $\Delta S_{M}(T)$ for a) the natural mineral and b) the synthetic sample of melanostibite under a magnetic field change of $1,2,3,4$, and $5 \mathrm{~T}$ (arrow denotes descending fields). 
From isothermal magnetization measurements around the magnetic phase transition, the Arrott plot can be constructed [15]. Isothermal magnetization data and Arrott plots for both the mineral and synthetic melanostibite are shown in Figure 4. As can be seen in Figure 4b and 4 $\mathrm{d}$ a positive slope in the entire data range and therefore a second order nature of the magnetic phase transition is evident for both the melanostibite mineral and synthetic sample (Banerjee criterion). Furthermore, the presented data feature a set of parallel straight lines around the transition temperature which indicate a mean-fieldlike behavior of the phase transition. For the mineral melanostibite, the behavior of the data is fairly linear as indicated by red line (linear fit) shown in Figure $4 \mathrm{~b}$. In contrast, some discrepancies presumably due to the presence of the secondary $\mathrm{MnFe}_{2} \mathrm{O}_{4}$ phase, were observed for the synthetic sample. The $\mathrm{MnFe}_{2} \mathrm{O}_{4}$ phase, as uncovered by our Rietveld analysis, is also evident in the isothermal magnetization data as a hysteresis background at the highest temperature of $288 \mathrm{~K}$ in Figure 4d. This is considerably above the transition temperature of the melanostibite main phase.

The magnetocaloric properties for the mineral are displayed in Figure 5a. Melanostibite undergoes a second-order phase transition, hence the peak $\Delta S_{M}^{p k}$ in the magnetic entropy change as function of temperature occurs at the phase transition temperature and is not shifted with increasing magnetic field changes. Nonetheless, the transition was found to be quite sharp and the magnetic entropy change $\Delta S_{M}$ is estimated to be $1.7 \mathrm{~J} / \mathrm{kgK}$ and 0.51 $\mathrm{J} / \mathrm{kgK}$ for magnetic field changes of $5 \mathrm{~T}$ and $1 \mathrm{~T}$, respectively. The magnetic entropy change for the synthetic sample can be seen in Figure 5 b. The magnetic entropy change for the synthetic sample under a magnetic field change of $5 \mathrm{~T}$ and $1 \mathrm{~T}$ was found to be $1.8 \mathrm{~J} / \mathrm{kgK}$ and $0.46 \mathrm{~J} / \mathrm{kgK}$, respectively. Hence, both samples show comparable values for the magnetic entropy change of the order of $2 \mathrm{~J} / \mathrm{kgK}$ for a magnetic field change of $5 \mathrm{~T}$. Interestingly, $\mathrm{Mn}_{2} \mathrm{FeSbO}_{6}$ crystallizes in a layered structure (c.f. Fig. 1) in which the inter-layer coupling of the $\mathrm{Mn}$ and $\mathrm{Fe} / \mathrm{Sb}$ layers is antiferromagnetic, whereas the intra-layer coupling is ferromagnetic. It is well know that the carrier doping and associated chemical pressure may change the inter-layer coupling towards a ferromagnetic behavior, e.g. in perovskite manganites [18]. Therefore, it is reasonable to expect that cation substitution could be employed to enhance the ferromagnetic interaction in $\mathrm{Mn}_{2} \mathrm{FeSbO}_{6}$, yielding improved magnetocaloric properties.

The magnetic field dependence of the magnetocaloric response is shown in Figure 6. In agreement with the work of Oesterreicher and Parker [3], a power 

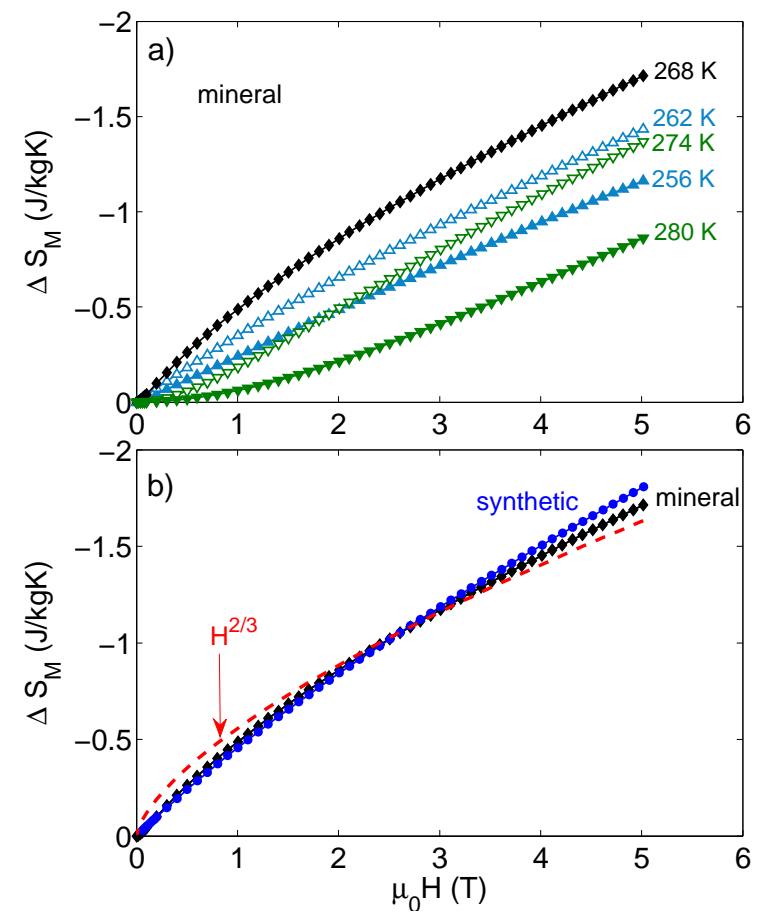

Figure 6: (Color online) a) Magnetic field dependence of the magnetic entropy change $\Delta S_{M}(H)$ for the natural mineral at different temperatures. b) $\Delta S_{M}(H)$ for the natural mineral $(\bullet)$ and synthetic $(\bullet)$ melanostibite at their respective transition temperature and the $H^{2 / 3}$ power law dependence (dashed lines).

law dependence for the magnetic entropy change $\Delta S \propto a H^{n}$ is observed. At the transition temperature the exponent $n$ for a system with well localized magnetic moments is expected to be aproximately $n=2 / 3$. In Figure $6 \mathrm{~b}$ the magnetic field dependence of the magnetic entropy change at the phase transition for the mineral $(268 \mathrm{~K})$ and synthetic $(264 \mathrm{~K})$ melanostibite as well as the $H^{2 / 3}$ power law are shown. It is evident that the experimental data at the transition temperature for mineral and synthetic $\mathrm{Mn}_{2} \mathrm{FeSbO}_{6}$ follow a $H^{2 / 3}$ dependence and the magnetic moments are localized.

Figure 7 depicts the magnetic entropy change $\Delta S_{M}$ for a $1 \mathrm{~T}$ field change derived from measurements of the specific heat and isothermal $M$ vs. $H$ curves. The $\Delta S_{M}$ curve from specific heat measurements was shifted with a constant offset to match the $\Delta S_{M}$ at $290 \mathrm{~K}$ obtained from $M$ vs. $H$ measurements and Equation 2. From a single $M$ vs. $T$ measurement in $1 \mathrm{~T}$ applied field $-d M / d T \times H$ was calculated. This quantity is of the same unit 


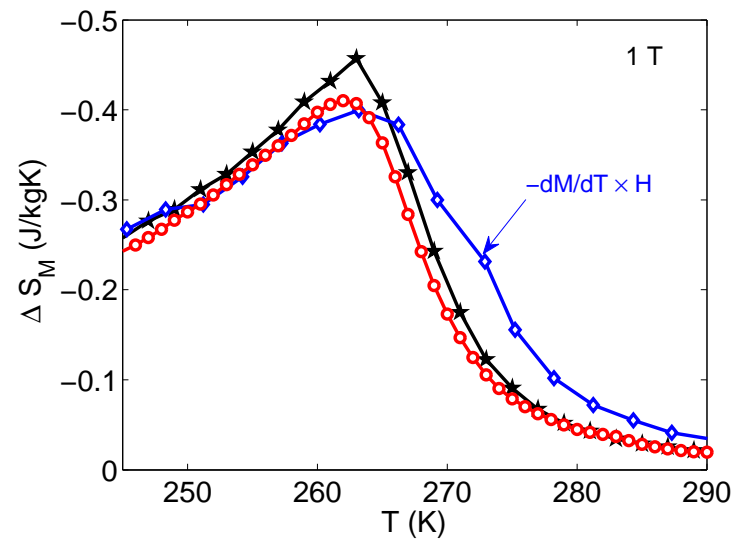

Figure 7: (Color online) The magnetic entropy change $\Delta S_{M}$ under $1 \mathrm{~T}$ field change as derived from specific heat ( $\circ)$ and $M$ vs. $H(\star) .-d M / d T \times H$ was derived from $M$ vs. $T$ in $1 \mathrm{~T}$ applied field $(\diamond)$.

as $\Delta S_{M}$. One can conclude that all three curves reproduce the qualitative behavior for the temperature dependence of the magnetic entropy change and only differ slightly in the absolute value for the $\Delta S_{M}$. It is interesting to note that a single $M$ vs. $T$ measurement at constant magnetic field gives an acceptable estimate of the magnetic entropy change for a second-order magnetocaloric material.

From the magnetic entropy change and the specific heat as function of temperature and magnetic field one can derive an estimate for the adiabatic temperature change. The magnetocaloric effect in terms of the change in temperature $\Delta T$ under a $1 \mathrm{~T}$ field change for the synthetic melanostibite sample can be estimated to

$$
\Delta T=-\Delta S_{M} \frac{T}{C_{P}(264 \mathrm{~K}, 1 \mathrm{~T})} \approx 0.2(\mathrm{~K}),
$$

where $C_{P}(264 \mathrm{~K}, 1 \mathrm{~T})=231 \mathrm{~J} / \mathrm{molK}$ is the specific heat measured at $264 \mathrm{~K}$ in $1 \mathrm{~T}$ applied field. An overview of the magnetocaloric properties of both mineral and synthetic $\mathrm{Mn}_{2} \mathrm{FeSbO}_{6}$ are listed in Table 1.

\section{Conlusions}

The magnetic properties of both natural single-crystal mineral and synthetic ceramic $\mathrm{Mn}_{2} \mathrm{FeSbO}_{6}$ with ilmenite-type structure have been investi- 
Table 1: Magnetic and structural properties for mineral and synthetic $\mathrm{Mn}_{2} \mathrm{FeSbO}_{6}$ (MFSO).

\begin{tabular}{|c|c|c|}
\hline Properties & mineral $\mathbf{M} \mathbf{M}_{2} \mathbf{F e S b O}$ & synthetic $\mathbf{M n}_{2} \mathbf{F e S b O} \mathbf{O}_{6}$ \\
\hline Mn:Fe:Sb & $2.03: 0.96: 0.99$ & $2.05: 0.91: 1.04$ \\
Space group & $\mathrm{R} \overline{3}$ & $\mathrm{R} \overline{3}$ \\
$a(\AA)$ & $5.226(1)$ & $5.237(1)$ \\
$c(\AA)$ & $14.325(2)$ & $14.349(2)$ \\
\hline$T_{N}(\mathrm{~K})$ & $268(2)$ & $264(2)$ \\
$\left.\Delta S_{M}^{P k}\right|_{5 \mathrm{~T}}(\mathrm{~J} / \mathrm{kgK})$ & 1.7 & 1.8 \\
$\left.\Delta S_{M}^{P k}\right|_{1 \mathrm{~T}}(\mathrm{~J} / \mathrm{kgK})$ & 0.51 & 0.46 \\
$\left.\Delta T\right|_{1 \mathrm{~T}}(\mathrm{~K})$ & - & 0.2 \\
\hline
\end{tabular}

gated. It was found that the natural mineral $\mathrm{Mn}_{2} \mathrm{FeSbO}_{6}$ undergoes a secondorder magnetic phase transition at $268 \mathrm{~K}$. The synthetic $\mathrm{Mn}_{2} \mathrm{FeSbO}_{6}$ orders at $264 \mathrm{~K}$. Both samples order ferrimagneticly and show comparable values for the magnetic entropy change $\Delta S_{M}$ on the order of $2 \mathrm{~J} / \mathrm{kgK}$ for $5 \mathrm{~T}$ and 0.5 $\mathrm{J} / \mathrm{kgK}$ for $1 \mathrm{~T}$ field change, respectively. The adiabatic temperature change for the synthetic $\mathrm{Mn}_{2} \mathrm{FeSbO}_{6}$ near room-temperature under $1 \mathrm{~T}$ field change was estimate to $\Delta T=0.2 \mathrm{~K}$. We believe that new functional magnetic materials with enhanced magnetocaloric properties based on $\mathrm{Mn}_{2} \mathrm{FeSbO}_{6}$ and its substitutions could be designed.

\section{Acknowledgments}

We thank the Swedish Energy Agency (STEM), Swedish Research Council (VR), the Göran Gustafsson Foundation, the Swedish Foundation for International Cooperation in Research and Higher Education (STINT), and the Russian Foundation for Basic Research for financial support. We are grateful to Dr. Henrik Skogby from the Swedish Museum of Natural History in Stockholm, Sweden, for providing melanostibite mineral.

\section{References}

[1] V. K. Pecharsky and K. A. Gschneidner, " $\mathrm{Gd}_{5}\left(\mathrm{Si}_{x} \mathrm{Ge}_{1-x}\right)_{4}$ : An extremum material," Advanced Materials 13, 683 (2001).

[2] N. H. Dung, Z. Q. Ou, L. Caron, L. Zhang, D. T. Cam Thanh, G. A. de Wijs, R. A. de Groot, K. H. J. Buschow, and E. Brück, "Mixed 
magnetism for refrigeration and energy conversion," Advanced Energy Materials 1, 1215 (2011).

[3] H. Oesterreicher and F. T. Parker, "Magnetic cooling near Curie temperatures above 300 K," J. Appl. Phys. 55, (22) 4334 (1984).

[4] L. J. Igelström, "Mineralogiska meddelanden - 18. Melanostibian, ett nytt antimonmineral från Sjögrufvan," GFF 14, 583 (1892).

[5] P. Moore, "Substitutions of the type $\mathrm{Sb}_{0.5}^{5+} \mathrm{Fe}_{0.5}^{3+}-\mathrm{Ti}^{4+}$; the crystal structure of melanostibite." Am. Min. 53(7-8), 1104 (1968).

[6] R. Mathieu, S. A. Ivanov, G. V. Bazuev, M. Hudl, P. Lazor, V. Solovyev, and P. Nordblad, "Magnetic order near $270 \mathrm{~K}$ in mineral and synthetic $\mathrm{Mn}_{2} \mathrm{FeSbO}_{6}$ ilmenite," Appl. Phys. Lett. 98, 202505 (2011).

[7] A. Rebello and R. Mahendiran, "Composition dependence of magnetocaloric effect in $\mathrm{Sm}_{1-x} \mathrm{Sr}_{x} \mathrm{MnO}_{3}$ (x=0.3-0.5)," Appl. Phys. Lett. 93, 232501 (2008).

[8] A. Rebello, V. B. Naik, and R. Mahendiran, "Large reversible magnetocaloric effect in $\mathrm{La}_{0.7-x} \mathrm{Pr}_{x} \mathrm{Ca}_{0.3} \mathrm{MnO}_{3}, "$ J. Appl. Phys. 110, 013906 (2011).

[9] H. Sakai, Y. Taguchi, and Y. Tokura, "Impact of Bicritical Fluctuation on Magnetocaloric Phenomena in Perovskite Manganites," J. Phys. Soc. Jpn. 78, 113708 (2009).

[10] T. J. Swoboda, R. C. Toole, and J. D. Vaughan, "New magnetic Compounds of the Ilmenite-type Structure," J. Phys. Chem. Solids 5, 293 (1958).

[11] G. V. Bazuev, B. G. Golovkin, L. N. V., N. I. Kadyrova, and Y. G. Zainulin, "High Pressure Synthesis and Polymorphism of Complex Oxides $\mathrm{Mn}_{2} \mathrm{BSbO}_{6}(\mathrm{~B}=\mathrm{Fe}, \mathrm{V}, \mathrm{Cr}, \mathrm{Ga}, \mathrm{Al})$," J. Solid State Chem. 124, 333 (1996).

[12] E. Warburg, "Magnetische Untersuchung I. Über einige Wirkungen der Coerzitivkraft (Nachweis der Hysteresiswärme)." Ann. Phys. 13, 141 (1881). 
[13] A. M. Tishin and Y. I. Spichkin, The Magnetocaloric Effect and its Applications, edited by J. M. D. Coey, D. R. Tilley, and D. R. Vij (IOP Publishing Ltd, 2003).

[14] N. A. de Oliveira and P. J. von Ranke, "Magnetocaloric effect around a magnetic phase transition," Phys. Rev. B 77, 214439 (2008).

[15] A. Arrott, "Criterion for Ferromagnetism from Observations of Magnetic Isotherms," Phys. Rev. 108, 1394 (1957).

[16] A. Arrott and J. E. Noakes, "Approximation equation of state for nickel near its critical temperature," Phys. Rev. Lett. 19, 786 (1967).

[17] S. Banerjee, "On a generalised approach to first and second order magnet transitions," Phys. Lett. 12, 16 (1964).

[18] T. Okuda, T. Kimura, H. Kuwahara, Y. Tomioka, A. Asamitsu, Y. Okimoto, E. Saitoh, and Y. Tokura, "Roles of orbital in magnetoelectronic properties of colossal magnetoresistive manganites," Materials Science and Engineering: B 63, 163 (1999). 